aimed to describe different service models and summarize evidence on impacts. Data from included publications were extracted and analysed narratively. Quality of included publications was assessed. Evidence on impact is presented according to each service objective.

Results Health-justice partnerships were diverse in their characteristics. Target populations focused on low income, vulnerable or underserved groups. Approaches to service coordination included co-location, referral, or integration of legal advisors into care teams and care pathways. The strongest evidence on impact was for increased access to legal assistance and improvements in individuals' financial and social circumstances. Despite this role in addressing social determinants of health, evidence was lacking on the wider issues of prevention and health inequalities. There was strong qualitative evidence for improvements in mental wellbeing, reflected in some quantitative research. Studies suggested positive outcomes in supporting healthcare professionals and contributing to high quality patient care, although findings on healthcare utilisation and costs were mixed.

Discussion This is the first international review on the delivery of health-justice partnerships, bringing together evidence from across the world to map current knowledge on service models and impacts. Integration of health and legal services aligns with policy priorities in both sectors, addressing social welfare legal issues which are leading causes of inequality and underlying determinants of poor health. The evidence supports health-justice partnerships as a means of improving access to justice for vulnerable groups and alleviating health-harming legal needs. Some key service objectives require a stronger knowledge base, while others remain un-evidenced. Cross-sector alignment and collaboration presents challenges, and further research is needed on successful delivery of integrated services. This review has informed a primary research study exploring implementation of health-justice partnerships in different settings across England to inform future policy and practice.

\section{P91 IMPACT OF THE STATUTORY CONCESSIONARY TRAVEL SCHEME ON BUS TRAVEL AMONG OLDER PEOPLE: A NATURAL EXPERIMENT FROM ENGLAND}

E Whitley*, P Craig, F Popham. Social and Public Health Sciences Unit, University of Glasgow, Glasgow, UK

\subsection{6/jech-2020-SSMabstracts. 183}

Background In the context of ageing populations worldwide, increasing numbers of older people are lonely, isolated and excluded, with serious implications for their health, cognitive and physical functioning. Access to good public transport can improve mobility, access to services and social participation among older adults. Policies that improve access and encourage the use of public transport are therefore potentially important in promoting healthy and successful ageing. Concessionary travel schemes for older people are in place in many countries but are under threat following the global financial crisis. Evidence regarding the success of these schemes in increasing activity and social participation is generally positive but based largely on qualitative or observational associations. In particular, robust evaluations of the schemes are limited by the lack of appropriate comparison groups as they generally represent a universal benefit.
Methods We use changes to the English statutory concessionary travel scheme over time, in particular the rising eligibility age from 2010 onwards, as a natural experiment to explore its impact on older people's travel. A difference-in-differencein-difference analysis of the annual National Travel Surveys (2002-2016) compares three age groups differentially affected by the eligibility criteria: (i) those aged 50-59 years who were never eligible for the scheme ( $\sim 2,500$ per year); (ii) those aged 60-64 years who were decreasingly eligible for the scheme from 2010 onwards (N 1,000 per year); and (iii) those aged 65-74 years who were consistently eligible for the scheme throughout the period of interest $(\mathrm{N} \sim 2,000$ per year). Results Compared with 50-59 year-olds, bus travel among 6074 year-olds increased year-on-year from 2002 to 2010, particularly from 2006 when the scheme offered free travel. The frequency of bus travel in both the older groups then fell following rises in eligibility age (annual change in the proportion traveling by bus at least weekly: $-2.9 \%(-4.1 \%,-1.7 \%)$ in 60 64 and 65-74 compared with 50-59 year-olds). Results were consistent across gender, occupation and urban/rurality.

Conclusion Our results indicate that access to, specifically, free travel increases bus use and access to services among older people, potentially improving mobility, social participation and health. However, the rising eligibility age in England has led to a reduction in bus travel in older people, including those not directly affected by the change, demonstrating that the positive impact of the concession goes beyond those who are eligible. Future work should explore the cost/benefit trade-off of this and similar schemes worldwide.

\section{P92 A MATCHED ANALYSIS OF THE IMPACT HAVING A CARER HAS ON AN INDIVIDUAL'S HEALTH AND SOCIAL CARE UTILISATION ACROSS FIVE SETTINGS OF CARE FOR ADULT RESIDENTS OF BARKING AND DAGENHAM}

1J Shand*, ${ }^{1} \mathrm{M}$ Gomes, ${ }^{2} \mathrm{~S}$ Morris. 'Department of Applied Health Research, UCL, London, UK; ${ }^{2}$ Department of Public Health and Primary Care, University of Cambridge, Cambridge, $U K$

\subsection{6/jech-2020-SSMabstracts. 184}

Background Across the UK today, an estimated 6.8 million people are carers, supporting friends and family who are older, disabled or seriously ill. The economic value of informal carers contributions is an estimated $£ 132$ billion a year. This is calculated by multiplying the total hours of care provided by carers (using responses from the 'Personal Social Services Survey of Adult Carers in England 2014-15' to determine numbers of carers and average hours of care provided) by the unit cost of an hour of replacement homecare for an adult $(£ 17.20)$. This assumes informal carers provide substitution for formal care. Reliance on carers appears to be increasing, with fewer people getting access to formal support as a result of local authority budget cuts.

This study aimed to understand if people with a carer have different levels of service use across different settings of care when compared to those who do not have a carer but have similar characteristics.

Methods A quantitative study using person-level data in Barking and Dagenham (B\&D), a London borough, to assess the impact of having a carer in terms of the differences in costweighted utilisation relative to a matched control group. Nearest neighbour matching was used, matching on age, 